VIII ${ }^{\text {èmes }}$ Journées Nationales Génie Civil - Génie Côtier, Compiègne, 7-9 septembre 2004

\title{
Modélisation du déferlement des vagues avec une méthode VOF sans reconstruction d'interface
}

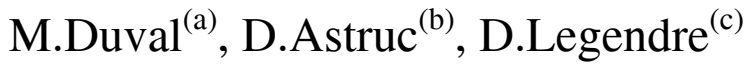 \\ ${ }^{(a)}$ Doctorante, ${ }^{(b)(c)}$ maîtres de conférences \\ Institut de mécanique des Fluides de Toulouse \\ UMR 5502 CNRS-INPT-UPS \\ Allée du Pr.C.Soula, 31400 Toulouse, France
}

\begin{abstract}
Résumé
L'objectif de ce travail est de tester la pertinence d'une méthode VOF sans reconstruction d'interface pour modéliser le déferlement des vagues. Tout d'abord, une onde de Stokes en eau profonde conduisant au déferlement plongeant est étudiée et montre la même dynamique que les simulations VOF avec reconstruction d'interface. La dissipation d'énergie est aussi étudiée. Ensuite, le déferlement d'une onde solitaire se propageant sur un récif immergé est testé. Les résultats sont en accord avec ceux obtenus avec la théorie potentielle jusqu'au point de déferlement, mais par la suite, le manque de résolution du maillage utilisé empêche le développement du jet.
\end{abstract}

\begin{abstract}
$\underline{\text { Abstract }}$
The relevance of a Navier Stokes VOF model without interface reconstruction for wave breaking modeling is investigated. First, a Stokes wave in deep water leading to a plunging breaker is performed and shows to the same breaking dynamics as interface-reconstructed computations. Energy dissipation is also investigated. Second, the breaking of a solitary wave over a submerged reef is computed. The results are similar to the potential flow computations in the early stage of the development of the breaker. However a lack in the grid resolution prevents a proper computation of the developed roller.
\end{abstract}

Mots-clés: Simulation numérique, méthode VOF, vague de Stokes, onde solitaire, déferlement.

\section{Introduction}

Le déferlement des ondes de surface a relc cu une attention particulière depuis ces dix dernières années, non seulement pour son intérêt fondamental, mais aussi pour son implication dans la dynamique côtière (forces induites sur les structures côtières, génération de spray, mise en suspension de sédiments,...), autant qu'en océanographie (interactions

air-océan.... $)^{1}$. Le déferlement des vagues est responsable de génération de turbulence et contrôle les flux de masse et de quantité de mouvement ou le taux de dissipation d'énergie. Les mesures expérimentales ${ }^{2}$ représentent une source indispensable de données pour analyser le déferlement des ondes de surface. Une alternative est la modélisation numérique. Les méthodes numériques peuvent être schématiquement divisées en trois groupes. Le premier rassemble les méthodes dans lesquelles le maillage est adapté ou se déforme pour suivre le mouvement de l'interface. On y retrouve la méthode d'intégrale de frontière $(\mathrm{BEM})^{3,4}$ par exemple, qui offre des solutions pour les déformations de la surface jusqu'à rupture de l'interface pour un écoulement potentiel, mais aussi des méthodes résolvant l'ensemble des équations de Navier-Stokes dans chaque phase qui satisfont de manière exacte les conditions 
de raccord à l'interface. De telles méthodes à maillage adaptatif, sont limitées à la première étape du déferlement, i.e. avant les grandes déformations de l'interface. Dans le second groupe de méthodes, l'écoulement est simulé avec un maillage fixe, sur lequel l'interface se déplace librement. Parmi ces méthodes, la plus populaire reste la méthode Volume Of Fluid (VOF), prenant son origine dans le travail pionnier de Hirt et Nichols ${ }^{5}$. La méthode VOF a été améliorée sous beaucoup d'aspects ces dernières années ${ }^{6}$ et utilisée pour simuler le déferlement des ondes de surface ${ }^{7,8}$. Les méthodes lagrangiennes forment un troisième groupe de méthodes qui permettent de s'affranchir de tout maillage. Parmi elles, la méthode Smoothed Particule Hydrodynamics $(\mathrm{SPH})^{15}$ à été utilisée pour des applications hydrauliques ${ }^{16}$. La méthode numérique utilisée pour cette étude ${ }^{9}$ est une méthode VOF dans laquelle aucun algorithme lagrangien n'est utilisé pour reconstruire l'interface. L'objectif de ce travail est de valider cette approche pour des simulations du déferlement des vagues. Deux situations physiques, une onde de Stokes en eau profonde et une onde solitaire se propageant sur un récif immergé, sont étudiées et les résultats comparés à des résultats numériques obtenus avec d'autres méthodes.

\section{$\underline{\text { 2.Modèle numérique }}$}

Comme pour les méthodes VOF usuelles, l'écoulement de deux phases incompressibles est gouverné par les équations de conservation suivantes:

$$
\left\{\begin{array}{l}
\nabla \cdot \underline{u}=0 \\
\frac{\partial \underline{\underline{u}}}{\partial t}+\nabla \cdot(\rho \underline{u \underline{u}})=-\nabla p+\nabla \cdot(T)+\rho \underline{g}+\left\langle 2 \sigma H \delta_{I} \underline{n}\right\rangle
\end{array}\right.
$$

où $\underline{u}$ et $p$ représentent les champs de vitesse et de pression, la masse volumique locale, $\mathrm{T}$ le tenseur des contraintes visqueuses, g la gravité, la tension interfaciale, $2 \mathrm{H}$ la courbure de la surface libre, $\delta_{I}$ une fonction de Dirac concentrée à l'interface et $\underline{n}$ le vecteur normal à la surface libre. <> représente un opérateur de moyenne spatiale. Le dernier terme dans l'équation de quantité de mouvement représente la force capillaire par unité de volume agissant sur les interfaces. L'introduction d'une fraction de volume $\mathrm{C}$ permet de définir un modèle à un fluide. $\mathrm{C}$ est définie dans tout l'écoulement, elle vaut 0 dans la phase liquide, 1 dans la phase gazeuse et possède une valeur intermédiaire dans les cellules traversées par la surface libre. Les propriétés physiques du fluide s'expriment en tout point de l'espace en fonction de C. Par exemple, la masse volumique et la viscosité dynamique (indice 1 pour le gaz et 2 pour le liquide):

$$
\left\{\begin{array}{l}
\rho=\rho_{1} C+(1-C) \rho_{2} \\
\mu=\mu_{1} C+(1-C) \mu_{2}
\end{array}\right.
$$

L'évolution de C est gouvernée par l'équation de conservation:

$$
\frac{\partial C}{\partial t}+\underline{u} \cdot \nabla C=0
$$

L'équation 3 est résolue en utilisant un schéma FCT avec limiteur de Flux de Zalesak ${ }^{11}$. Ce schéma est implémenté ici sous sa forme monodimentionnelle pour laquelle l'équation 3 est projetée suivant les trois directions de l'espace, ce qui correpond à trois problèmes 
monodimentionnels qui sont résolus successivement, de manière découplée de façon à minimiser les distorsions de la surface libre. Pour l'équation 1, les opérateurs sont discrétisés en espace en utilisant une méthode de volumes finis du second ordre.

L'évolution temporelle s'appuie sur un algorithme semi-implicite de Runge-Kutta/CrankNicolson du second ordre. L'incompressibilité est satisfaite à la fin de chaque pas de temps, par une inversion directe de l'équation de Poisson 2D pour un potentiel auxiliaire. Le terme de force capillaire est résolu en utilisant une formulation continue ${ }^{10}$.

Une limite de la méthode, comme la plupart des méthodes VOF, est qu'elle ne prend pas en compte les forces physiques dont la longueur caractéristique d'action est inférieure à la taille de la maille. Dans notre étude aucun modèle de sous grille n'est introduit, c'est à dire que l'on ne modélise pas la turbulence par exemple. Cependant, l'approche sans reconstruction d'interface permet la modélisation de région de mélange à des échelles inférieures à celle de la maille.

\section{Deferlement d'une onde de Stokes en eau profonde}

\subsection{Configuration physique et numérique}

Afin de tester la capacité de notre code numérique (sans reconstruction d'interface) pour simuler le déferlement plongeant, la configuration proposée par Chen et coll ${ }^{7}$ a été reprise. La condition initiale est une onde de Stokes du troisième ordre ${ }^{12}$, d'amplitude $a$ et de longueur d'onde , le champ de vitesses est implémenté à partir de la théorie potentielle dans la phase liquide et est initialement au repos dans la phase gazeuse. Le problème physique est contrôlé par cinq nombres sans dimention: le nombre de Reynolds: $\operatorname{Re}=\rho_{2} g^{1 / 2} \lambda^{3 / 2} / \mu_{1}=10^{4}$, le nombre de Bond: $B=\rho_{2} g^{\lambda^{2}} / \sigma=10^{4}$, la cambrure de la vague $\gamma=2 \pi a / \lambda=0.55$, les rapports entre les masses volumiques $\overline{\rho=} \rho_{1} / \rho_{2}=0.01$ et entre les viscosités $\bar{\mu}=\mu_{1} / \mu_{2}=0.4$.

On remarque que les propriétés physiques des deux fluides choisis par Chen et coll. ${ }^{7}$ ne sont pas les rapports réels entre l'eau et l'air. En eau profonde, les vagues déferlent sous la force du vent, les interactions avec des courants ou avec d'autres vagues. Dans notre étude, la condition initiale est instable, c'est pourquoi l'onde déferle. Les simulations sont réalisées dans un domaine carré de côté la longueur d'onde, sur un maillage 200x200 non-uniforme. Le maillage est uniforme suivant l'axe horizontal avec un pas $\Delta x=510^{-3} \lambda$.

Sur l'axe vertical, une région au centre est maillée uniformément où la taille des mailles est $\Delta z=1,6710^{-3} \lambda$. Elle est entourée de deux régions ayant une taille de maille décroissant vers le haut et le bas du domaine de calcul. Une condition de périodicité est appliquée sur les frontières latérales et de symétrie en haut et en bas du domaine. Des tests réalisés ont montré que la condition aux limites au fond n'avait pas d'influence sur la dynamique du déferlement. Ces simulations ont été réalisées sur un Pentium IV à 2,4 GHz et le temps CPU de ces calculs est de 11,1 secondes par pas de temps. Les résultats seront présentés dans le repère en translation à la vitesse de phase de l'onde par rapport au repère fixe, sous forme adimentionnée, en choisissant pour échelle caractéristique de longueur, et de temps, $(/ \mathrm{g})^{1 / 2}$. 


\subsection{Résultats}

\subsubsection{Dynamique du déferlement}

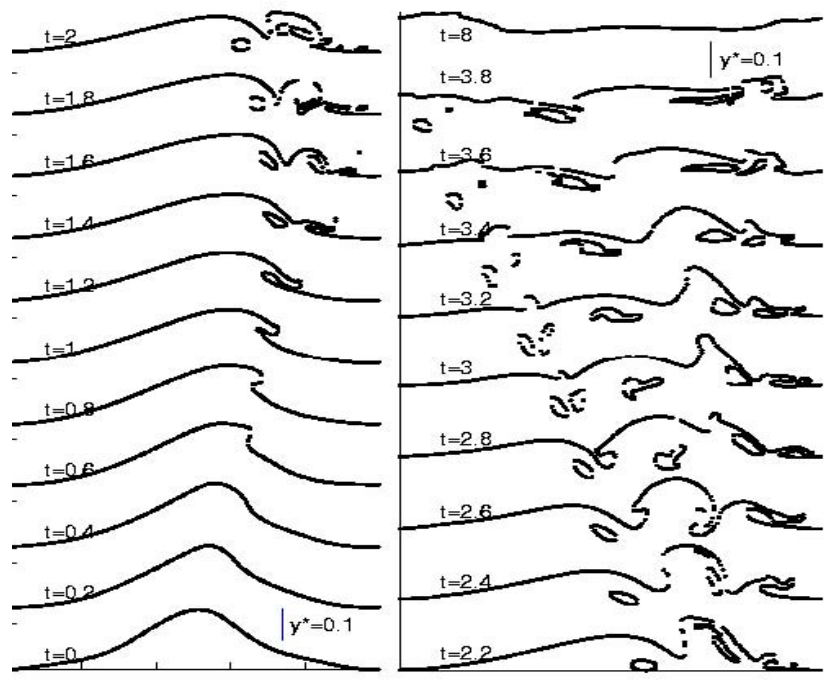

Figure 1: Profil de la surface libre $(C=0,5)$ à différents instants de l'évolution de l'onde de Stockes

Sur la figure 1, sont représentés les profils de la surface libre obtenus avec notre méthode numérique à différents instants. Tout d'abord, la crête de la vague se raidit jusqu'à devenir verticale, un jet de liquide se forme alors, grossit et tombe à l'avant de la vague sous l'influence de la gravité, dans un mouvement de renversement. Il s'ensuit l'impact du jet qui cause l'entraînement d'air et l'éjection d'un jet secondaire. Ce jet secondaire grossit et évolue à son tour en un mouvement de renversement. On peut observer quatre cycles de jet secondaire successifs. La dynamique du déferlement est identique à celle décrite par Chen et coll. .

\subsubsection{Energie}

L'énergie mécanique totale $\mathrm{E}$ est définie comme la somme des énergies cinétique Ec, potentielle Ep et capillaire. Une estimation de l'ordre de grandeur des énergies montre que l'énergie capillaire est cent fois inférieure aux deux autres, elle peut donc être négligée. La figure 2 montre que l'énergie totale diminue avec le temps. Tout d'abord, elle est peu dissipée d'ol'u une faible décroissance jusqu'à l'impact du jet à l'avant de la vague. L'approximation de conservation de l'énergie totale peut rester valable dans cette première phase du déferlement. Ensuite, sa décroissance est plus rapide et proche de $\mathrm{t}^{-1 / 4}$. A la fin de la simulation l'énergie potentielle a disparu, en effet, la phase liquide retrouve son niveau de repos. On trouve qu'environ 97\% (5\% dans le repère de la vague) de l'énergie mécanique est dissipée par le processus de déferlement. Ce résultat semble raisonnable en comparaison avec les simulations de Chen et coll. ${ }^{7}$ pour lesquelles $90 \%$ de l'énergie mécanique est dissipée. 

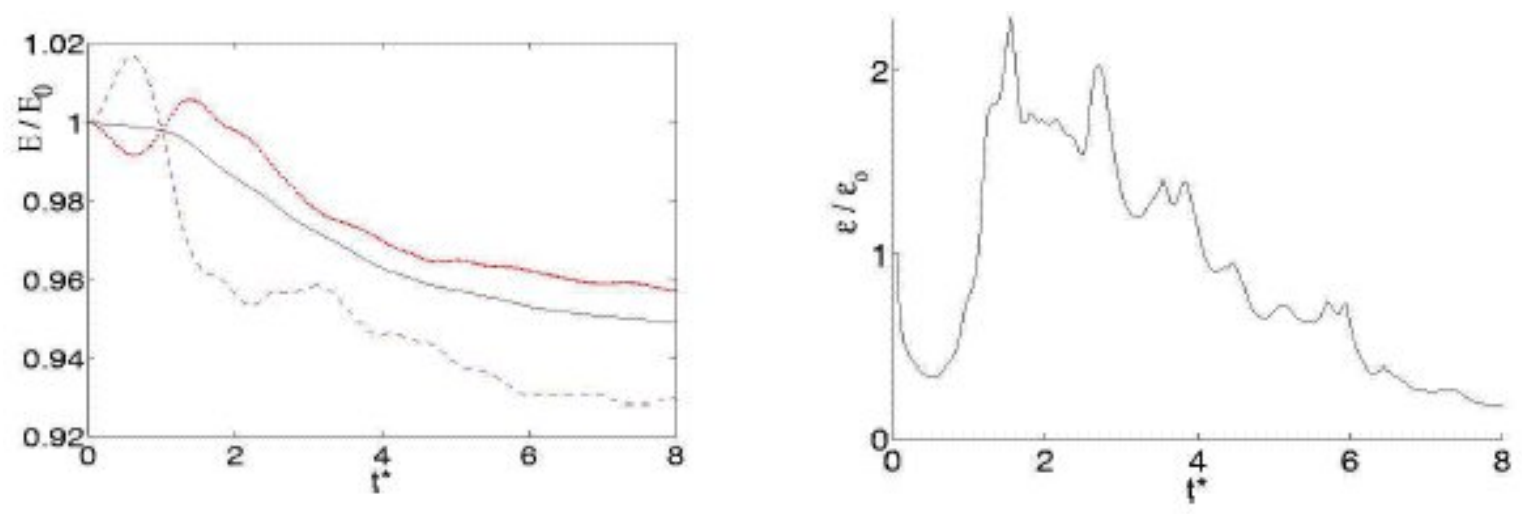

Figure 2: Evolution temporelle des énergies normalisées par leurs valeurs initiales: $E / E_{0}(-)$ avec $E_{0}=0,7321 \mathrm{Jm}^{-1}, E_{p} / E_{p 0}(-)$ avec $E_{p o}=0,2122 \mathrm{Jm}^{-1}$ et $E_{c} / E_{c o}($.$) avec E c o=0,5199 \mathrm{Jm}^{-1}$

Figure 3: Evolution temporelle du taux de dissipation d'énergie adimentionné normalisé par sa valeur initiale $0=5,227010^{-3} \mathrm{Jm}^{-1} \mathrm{~s}^{-1}$

La figure 2 montre aussi l'évolution des énergies Ep et Ec. Ep augmente alors que Ec diminue jusqu'au déferlement. Ep décroît pendant le processus de renversement jusqu'à l'impact du jet $\left(t^{*}=1,25\right)$ atteignant un minimum alors que Ec atteint son maximum. On observe qu'à chaque augmentation de Ep, correspond une diminution de Ec et vice versa. Il y a un transfert entre les deux énergies pendant les cycles de jets secondaires, comme pour le rebond d'une balle sur une surface rigide.

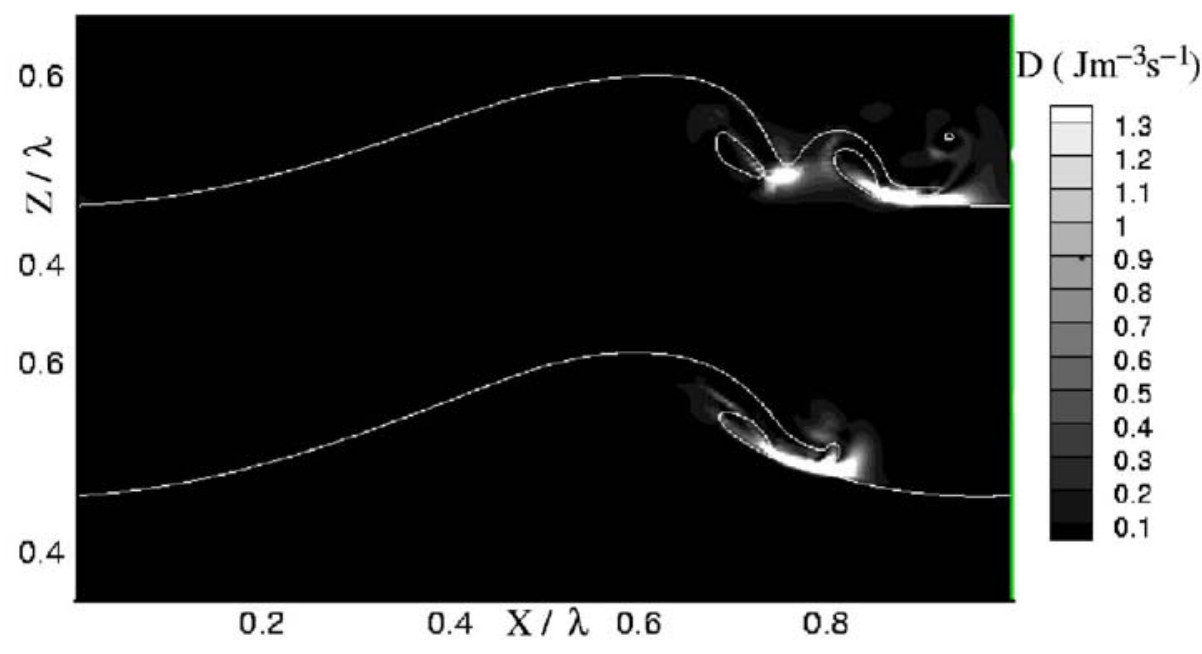

Figure 4: Densité de taux de dissipation d'énergie D dans le domaine $\left(\mathrm{J}_{m}{ }^{-3} \mathrm{~S}^{-1}\right)$ à $t^{*}=1,25$ en haut et $t^{*}=1,55$ en dessous

Le taux de dissipation d'énergie obtenu par intégration sur le domaine complet Figure 3.), décroît jusqu'à l'instant de déferlement et augmente significativement pendant le processus de renversement, atteignant un maximum local de 1,81 fois sa valeur initiale à l'impact du jet. Cette croissance se poursuit jusqu'à l'impact du premier jet secondaire où le taux de dissipation vaut 2,3 fois sa valeur initiale. Le taux de dissipation d'énergie décroît ensuite avec différents pics correspondant aux différents impacts des jets secondaires. On peut 
aussi remarquer (Figure. 4) que l'énergie est localement dissipée autour des points d'impact du jet et des jets secondaires. Ce processus local de dissipation se prolonge dans le temps.

\section{Onde solitaire déferlant sur un récif immergé}

\subsection{Configuration physique et numérique}

La configuration de Yasuda et coll. ${ }^{13}$ est utilisée pour étudier une onde solitaire déferlant sur un récif immergé. La condition initiale (forme, champ de vitesse et de pression) est une onde solitaire du neuvième ordre proposée par Fenton ${ }^{14}$. Les rapports suivants sont choisis pour donner a priori un déferlement de type plongeant: $\mathrm{R} / \mathrm{h}=0,6$ et $\mathrm{a} / \mathrm{h}=0,5$, où a représente l'amplitude de la vague, $\mathrm{R}$ la hauteur de récif et $\mathrm{h}$ la profondeur. Les propriétés physiques de l'air et de l'eau sont respectées dans nos simulations. Les calculs sont réalisés dans un domaine rectangulaire, de $20 \mathrm{~m}$ de long et de $1,5 \mathrm{~m}$ de haut. Un récif rectangulaire de $0,26 \mathrm{~m}$ de hauteur est placé à $15 \mathrm{~m}$ de la frontière gauche et l'onde solitaire initiale est centrée à 11,5 $\mathrm{m}$. Le maillage est non uniforme suivant les deux axes, mais une région centrée sur le récif où la vague doit déferler, est maillée uniformément avec $\Delta x=0,01 \mathrm{~m}$ et $\Delta z=0,005 \mathrm{~m}$. Un pas de temps constant est imposé pour les simulations $\Delta t=510^{-3} \mathrm{~s}$

\subsection{Résultats}

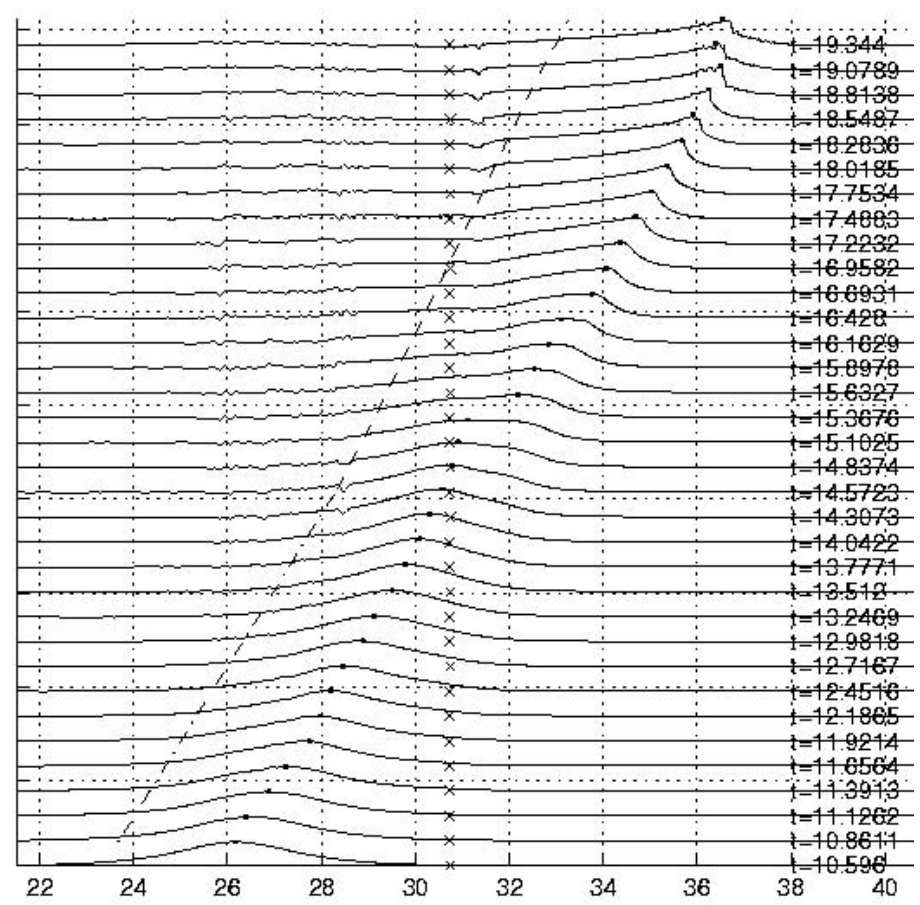

Figure 5: Profils de la surface libre $(C=0.5,-)$ d'une onde solitaire se propageant vers un récif immergé; position du récif $(X)$, élevation maximum de la surface libre: (.), vitesse de phase théorique de l'onde solitaire fonction de profondeur: (--)

Tout d'abord (Figure. 5), l'onde solitaire se propage sans déformation. Lorsqu'elle atteint le récif, la crête commence à se raidir. On peut observer sur la surface arrière de la vague, une déformation pouvant être associée à l'adaptation de la vague à la nouvelle profondeur du domaine. L'onl-de continue de se raidir jusqu'au point de déferlement, lorsque la face avant de 
le crête devient verticale. Un jet semble être éjecté mais la vague dégénère en un bouillonnement. L'événement observé ressemble plus à un déferlement de type glissant qu'à un déferlement plongeant qui aurait dû être observé ${ }^{13}$. La dissymétrisation de la vague peut s'expliquer par la présence du récif. En effet, la propagation de la vague à deux profondeurs différentes cause une réfraction de l'onde, caractérisée par une augmentation de son amplitude et l'instabilité de la vague.

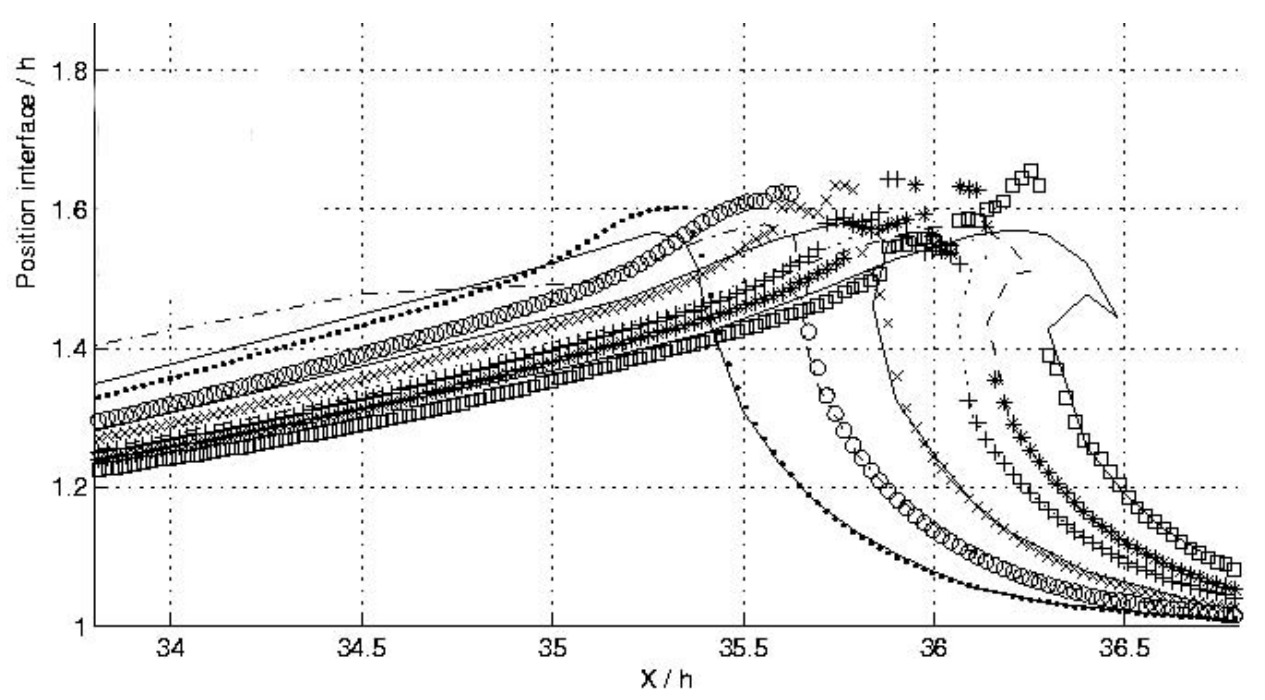

Figure 6:Superposition de profils de la surface libre à différents instants adimentionnés par $(\mathrm{h} / \mathrm{g})^{1 / 2}$ issus de nos simulations Navier-Stokes $(C=0,5)$ et de la théorie potentielle ${ }^{13}$; théorie potentielle, de gauche à droite: $t^{*}=17,8107(-), t^{*}=18,0209(-),. t^{*}=18,2024(-), t^{*}=18,3720(),. t^{*}=18,4599(--)$ et $t^{*}=18,6098(-) ;$ simulations VOF, de gauche à droite: $t^{*}=17,8107\left(\mathrm{)}, t^{*}=18,0209(\mathrm{)}), t^{*}=18,2024(\mathrm{X}), t^{*}=18,3720(+), t^{*}=18,4599(*)\right.$ et $t^{*}=18,6098()$

Afin de comparer nos résultats avec la théorie potentielle ${ }^{13}$, la figure 6 montre la dynamique de la surface libre à différents instants issus de nos simulations et de celles de Yasuda et coll. ${ }^{13}$ Qualitativement, on observe que les profils sont très similaires avant le point de déferlement. Ils se superposent parfaitement à l'avant et à l'arrière de la crête. Cependant, des différences apparaissent sur la hauteur de la vague (maximum 5,46\% par rapport à la théorie potentielle) liées à la présence de vorticité sur une interface courbe. On observe des oscillations à la place de l'éjection d'un jet. La résolution utilisées pour les simulations semble être trop grossière pour capturer correctement la deuxième phase du déferlement. Une résolution identique à celle utilisée dans les simulations avec l'onde de Stokes semble être nécessaire. Les simulations ont été réalisées sur un Pentium IV à 2,4 GHz et le temps CPU de ces simulations est d'environ 7,5 secondes par pas de temps.

\section{Conclusion}

La pertinence d'une méthode VOF sans reconstruction d'interface pour modéliser le déferlement en eau profonde et peu profonde est testée dans ce travail. Les résultats des simulations d'une onde de Stokes en eau profonde sont présentés et sont en assez bon accord dans la dynamique de la surface libre avec les résultats issus d'une méthode VOF avec reconstruction d'interface ${ }^{7}$. Des régions de mélange ont pû être observées, leur dynamique reste à valider. L'évolution des énergies a aussi été étudiée. La méthode a également été utilisée pour simuler le déferlement d'une onde solitaire se propageant sur un récif immergé. La dynamique du déferlement semble être modélisée avec précision dans la première partie de 
l'évolution de l'onde, mais les simulations manquent de raffinement pour la suite du processus de déferlement. Dans la perspective de modéliser des écoulements réalistes, l'intégration d'un modèle de sous-maille devra être envisagée.

\section{Remerciement}

Nous remercions le programme PATOM du CNRS ainsi que le programme CALMIP.

\section{Références}

1.Melville, W. K. (1996). The role of surface-wave breaking in air sea interaction. Ann. Rev. Fluid. Mech.,28, 279-321.

2.Bonmarin, P. (1989). Geometric properties of deep-waterbreaking wave. J. Fluid Mech., 209, 405-433.

3.Longuet-Higgins, M. S. and Cokelet, E. D. (1976). The deformation of steep surface waves on water I. A numerical method of computation. Proc. R. Soc. London\}, Ser. A, 350, $1-26$.

4.Longuet-Higgins, M. S. and Cokelet, E. D.(1978). The deformation of steep surface waves on water II. Growth of normal-mode instabilities. Proc. R. Soc. London\}, Ser. A, 364,1-28.

5.Hirt, C.W. and Nichols, B.D. (1981). Volume of fluid (VOF) method for the dynamics of free boundaries. J. Comput. Phys., 39, 201.

6.Scardovelli, R. and Zaleski, S. (1999). Direct numerical simulation of free-surface and interfacial flow. Annu. Rev. Fluid Mech., 31, 542.

7.Chen, G., Kharif, C., Zalesky, S., and Lie, J. (1997). Two-dimentional Navier Stokes simulation of breaking wave. Itextsl\{Phys. Fluid\}, 11, 121-133.

8.Abadie, S., Caltagironne, J.P. and Watremez, P. (1998). Mécanisme de génération du jet secondaire dans un déferlement plongeant. C. R. Acad. Sci. Paris \}, Ser. IIb, 326, 553-559.

9.Benkenida, A. and Magnaudet, J. (1999). Une méthode de simulation d'écoulements diphasiques sans reconstruction d'interface. C. R. Acad. Sci. Paris\}, Ser. II.

10.Brackbill, J. U., Kothe, D. B. and Zemach, C. (1992). A continuum method for modeling surface tension. J. Comput. Phys., 100, 335.

11.Zalesak, S. T.(1979). Fully multidimensional flux-corrected transport algorithm for fluids. J. Comput. Phys., 31, 335.

12.Lamb, H.(1932). Hydrodynamics. Cambridge University Press, Sect 250.

13. Yasuda, T., Mutsuda, H., and Mizutani, N. (1997). Kinetics of overturning solitary waves and their relations to breaker types. Coastal Eng., 29, 317-346. 271.

14.Fenton, J. (1972). A ninth-order solution for the solitary wave. J. Fluid Mech., 53, 257-

15.Monaghan, J. J. (1994). Simulating free surface flows with SPH. J. Comput. Phys., 110, 399-406.

16.Violot, D., Issa, R. (2003). La méthode SPH appliquée à l'hydraulique. Une méthode lagrangienne aux applications variées. Rev. Europ. Elmts. Finis,

$12, \mathrm{~N}^{\circ} 2-3 / 2003,171-190$. 